\title{
A 16 year old boy with chest pain
}

In the online version of this Endgames article (BMJ

2014;349:g6172, doi:10.1136/bmj.g6172), the acknowledgement

Cite this as: BMJ 2014;349:g7253

should have been to Lucia Gavalova, not Lucia Gavilova. The

๑ BMJ Publishing Group Ltd 2014

authors apologise for this misspelling. 